\title{
Microfluidic Impedance Biosensor Chip with DNA- Based Self-Assembled Monolayers for Label-Free Detection of Cardiac Biomarker Troponin $I^{+}$
}

\author{
Khaled Alsabbagh ${ }^{1}$, Tim Hornung ${ }^{1}$, Achim Voigt ${ }^{1}$, Sahba Sadir ${ }^{2}$, Taleieh Rajabi ${ }^{1}$ and Kerstin \\ Länge 1 ,* \\ 1 Institute of Microstructure Technology, Karlsruhe Institute of Technology, Hermann-von-Helmholtz-Platz \\ 1, 76344 Eggenstein-Leopoldshafen, Germany; \\ 2 Institute for Micro Process Engineering, Karlsruhe Institute of Technology, Hermann-von-Helmholtz-Platz \\ 1, 76344 Eggenstein-Leopoldshafen, Germany; \\ * Correspondence: kerstin.laenge@kit.edu \\ + Presented at the 1st International Electronic Conference on Biosensors, 2-17 November 2020; Available \\ online: https://iecb2020.sciforum.net/.
}

Received: date; Accepted: date; Published: date

\begin{abstract}
A microfluidic chip for electrochemical impedance spectroscopy (EIS) is presented as biosensor for the detection of cardiac troponin I (cTnI). Troponin I is one of the most specific diagnostic serum biomarkers for myocardial infarction. As impedimetric biosensors allow direct and label-free analyte detection, they are particularly suitable for fast biomarker detection. This is essential in the diagnosis of cardiac infarctions to enable an early treatment promoting a positive outcome. The microfluidic impedance biosensor chip presented here consists of a microscope glass slide serving as base plate, sputtered electrodes, and a polydimethylsiloxane (PDMS) microchannel. Electrode functionalization protocols were developed considering a low initial impedance in addition to analyte-specific binding by corresponding antibodies and reduction of non-specific protein adsorption to prevent false-positive signals. Reagents tested for self-assembled monolayers (SAMs) on gold electrodes included thiolated hydrocarbons and thiolated oligonucleotides, where SAMs based on the latter showed a better performance. The corresponding antibody (anti-cTnI) was covalently coupled on the SAM using carbodiimide chemistry. The PDMS microchannel was bonded on the glass slide with the functionalized electrodes, and the completed microfluidic impedance biosensor chip was connected to the readout system. Sampling and measurement took only a few minutes. Application of a human serum albumin (HSA) sample, $1000 \mathrm{ng} / \mathrm{mL}$, led to negligible signal changes, while application of a troponin I sample, $1 \mathrm{ng} / \mathrm{mL}$, led to a significant signal shift in the Nyquist plot. The results are promising regarding specific detection of clinically relevant concentrations of cardiac markers with the newly developed impedance biosensor chip.
\end{abstract}

Keywords: biosensor; cardiac troponin I; single-strand DNA; electrochemical impedance spectroscopy; label-free; microfluidic chip; self-assembled monolayers

\section{Introduction}

According to the World Health Organization (WHO), cardiovascular diseases (CVDs) are the leading cause of death worldwide. In 2016, 85\% of the people who died of CVD suffered a stroke or a heart attack. One way to reduce these numbers is to increase the survival rate by starting the treatment as early as possible, which requires an early diagnosis [1,2]. Diagnosis criteria of a heart attack (myocardial infarction) include the detection of biomarkers in blood. Particularly a concentration increase of cardiac troponins I and $\mathrm{T}(\mathrm{cTnI}, \mathrm{cTnT})$ indicate myocardial necrosis with 
normal levels being $<0.5-2.0 \mathrm{ng} / \mathrm{mL}$ (lab-specific) and $<0.1 \mathrm{ng} / \mathrm{mL}$, respectively, and a factor increase of up to 40 -fold and 40 to 60 -fold, respectively, in case of an infarction [3,4].

Label-free biosensors allow direct detection of analyte molecules and, hence, offer a tool for fast detection of biomarkers. Particularly electrochemical biosensors offer advantages here, as they can be fabricated cost-effectively, array-compatible, and customized in a comparatively easy way. A (micro-)fluidic channel can easily be made separately, e.g., from polydimethylsiloxane (PDMS), and then connected to the sensor unit. The requirements for biosensing layers include both the capability of analyte-specific binding and the minimization of non-specific binding. The latter is particularly important for label-free biosensors to avoid false-positive results. Electrochemical biosensors include impedimetric biosensors which measure the impedance, i.e., the opposition a circuit presents to a current when an alternating voltage is applied. Sensing layers of biosensors using electrochemical impedance spectroscopy (EIS) must also allow charge transfer to the electrode for the transduction principle to work [5-7].

Electrodes of impedance biosensors are typically made of gold. A well-established procedure for the introduction of functional groups on this material is to use suitably substituted thiols forming self-assembled monolayers (SAMs). Thiols with aliphatic hydrocarbon spacer of sufficient chain length result in well-defined and stable SAMs of high density. The brush-like structure of such layers make it possible to effectively reduce non-specific protein adsorption on the underlying gold surface. However, such SAMs may result in insulating layers, hindering the charge transfer required for the EIS. The use of aromatic hydrocarbons increases the layer's permeability for charge transfer, but nonspecific protein adsorption in the subsequent measurements may also increase because of a reduced density of the layer. Conductive polymers would offer an alternative, but are often linked with coating procedures more complex than wet chemistry $[5,6]$.

Thiolated single-strand DNA (ssDNA) oligomers, on the other hand, can be packed densely on the gold surface by wet chemistry methods similar to the thiolated hydrocarbons. Co-immobilization of thiolated ssDNA with thiolated hydrocarbons may be recommended to improve the integrity of the brush-like structure. The negatively charged backbone of the DNA oligomers - resulting from the composition of alternating sugar (deoxyribose) and phosphate groups-promises lower initial impedance values and, hence, permeability for charge transfer events. The immobilized ssDNA can be used directly as probe for DNA detection, but it can also be hybridized with ssDNA carrying functional groups for coupling analyte-specific capture molecules, such as antibodies $[5,6,8]$.

In this work, we introduce an own design for a microfluidic impedance biosensor chip. The base plate is a microscope glass slide carrying the gold electrodes, while the microfluidic channel is added as a PDMS component. Biosensing layers used thiol-SAMs based on both aromatic hydrocarbons and DNA, where the latter showed best performance regarding suppression of non-specific binding of human serum albumin (HSA) and specific binding of the cardiac marker troponin I.

\section{Materials and Methods}

\subsection{Fabrication of the Microfluidic Impedance Biosensor Chip}

\subsubsection{Base Plate and Electrode Sputtering}

Standard microscope glass slides were used as base plates for the impedance biosensor chips. They were first cleaned with a detergent solution, brushed with a toothbrush, and rinsed with water. Then, they were sonicated with bi-distilled water for $5 \mathrm{~min}$ using an ultrasonic cleaning bath. Finally, they were rinsed with filtered 2-proponol and blown dry with filtered nitrogen gas; the filter pore size was $0.2 \mu \mathrm{m}$ in both cases.

A parylene $C$ (poly(2-chloro-p-xylylene)) layer with a thickness of $0.1 \mu \mathrm{m}$ was applied on the cleaned glass slides as adhesion layer (SCS Labcoter ${ }^{\circledR}$ 1, PDS 2010, Specialty Coating Systems) [9]. Working and counter electrodes made of gold were applied on the parylene C-coated glass slides by using a corresponding mask made of steel and a sputtering system (Balzers MED 010). The gold was sputtered at approx. 0.06 mbar and a current of $30 \mathrm{~mA}$ with the time set to $15 \mathrm{~min}$, which led to a 
thickness in the range 15-35 $\mathrm{nm}$. The ratio of the areas of working electrode to counter electrode was approximately 1:100. A microscope glass slide with sputtered electrodes is shown in Figure 1a. Conducting paths led from the electrodes to the edge of the glass slide, where they formed contact pads for the connection to the measurement setup (see Section 2.3.1). A reference electrode was not included in this work, but can be included, if required (Figure 1b).

(a)

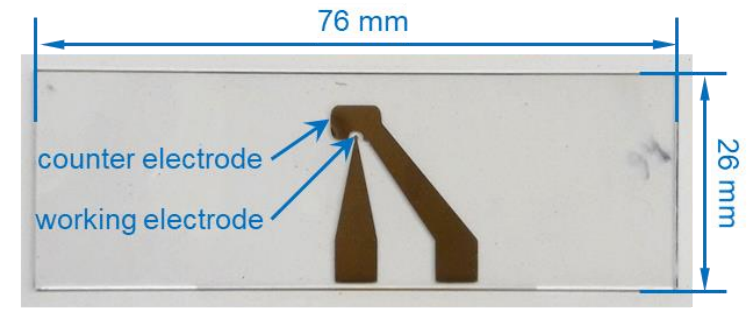

(b)

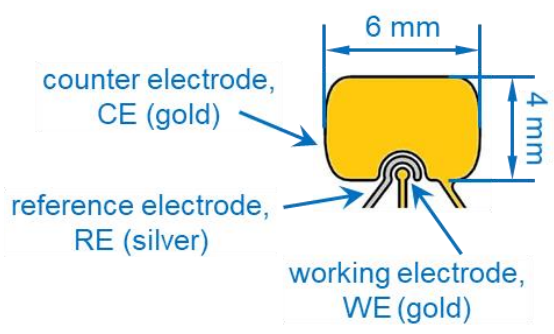

Figure 1. (a) Microscope glass slide with sputtered electrodes. (b) Detailed view of working and counter electrode, including a potential reference electrode.

\subsubsection{Microfluidic Channel Fabrication and Connecting}

The microfluidic channel was formed from PDMS. A mixture of PDMS base and curing agent (Sylgard ${ }^{\mathrm{TM}}$ 184, Dow) was casted into a milled form made of polymethyl methacrylate (PMMA) and incubated at $70^{\circ} \mathrm{C}$ for $2 \mathrm{~h}$. To combine the PDMS channel with the impedance biosensor chip carrying the electrodes, both parts were plasma-activated and assembled with light pressure. A picture of the combined parts is shown in Figure 2. If the electrodes have been chemically modified prior to bonding the PDMS channel (see Section 2.2), the respective parts were covered by impermeable polystyrene pieces.

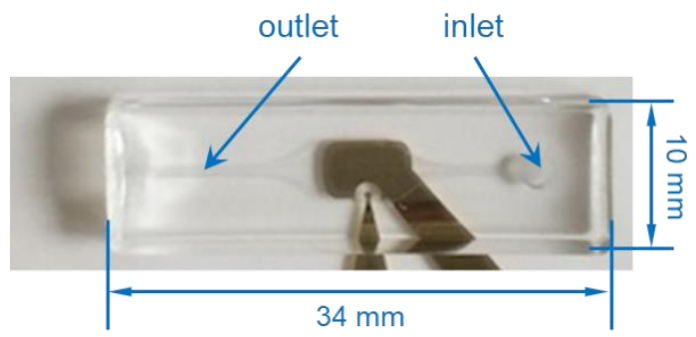

Figure 2. Microfluidic PDMS channel bonded on the impedance biosensor chip to deliver samples across the electrodes. The channel height was $0.2 \mathrm{~mm}$ leading to a channel volume below $10 \mu \mathrm{L}$.

\subsection{Surface Functionalization}

\subsubsection{Application of Thiol-SAMs with Hydrocarbon Spacer, Antibody Immobilization}

For SAM testing the electrodes were plasma activated and a drop of $10 \mu \mathrm{L}$ containing $50 \mathrm{mM} 4$ mercaptobenzoic acid or 1,4-benzenedithiol was pipetted on both working and counter electrodes. After incubation overnight at ambient temperature, the electrodes were rinsed with ethanol and the chip assembled with the PDMS channel (see Section 2.1.2).

For antibody immobilization the electrodes were plasma activated and a drop of $0.05 \mu \mathrm{L}$ containing $20 \mathrm{mM}$ 4-mercaptobenzoic acid dissolved in ethanol was pipetted on the working electrode. After $24 \mathrm{~h}$ of incubation at $6{ }^{\circ} \mathrm{C}$ the chip was rinsed with ethanol. After another plasma activation of the counter electrode, during which the working electrode was covered with a piece of polystyrene, $10 \mu \mathrm{L}$ of a solution of $20 \mathrm{mM}$ 1,4-benzenedithiol dissolved in ethanol was pipetted on the working electrode and incubated overnight at $6{ }^{\circ} \mathrm{C}$. After rinsing with ethanol and drying, $10 \mu \mathrm{L}$ 
of a freshly prepared aqueous solution containing $0.05 \mathrm{M}$ N-hydroxysuccinimide (NHS) and $0.2 \mathrm{M} 1-$ ethyl-3-(3-dimethylaminopropyl)carbodiimide (EDC) was pipetted on the electrodes. The mixture was supposed to react only with the carboxyl groups of the 4-mercaptobenzoic acid SAM on the working electrode resulting in an active ester [10]. After $30 \mathrm{~min}$ of incubation and rinsing with phosphate buffered saline (PBS), $0.05 \mu \mathrm{l}$ of an anti-troponin I solution, diluted with PBS to $5 \mu \mathrm{g} / \mathrm{mL}$, was pipetted on the working electrode. After $30 \mathrm{~min}$ of incubation and rinsing with PBS, $0.05 \mu \mathrm{l}$ of an aqueous solution of ethanolamine hydrochloride, $1 \mathrm{M}, \mathrm{pH}=8.5$, was pipetted on the working electrode for de-activation of potentially available still reactive active ester groups [10]. Finally, the impedance biosensor chip was thoroughly rinsed with PBS, dried, and assembled with the PDMS microfluidic channel (see Section 2.1.2).

\subsubsection{Application of Thiol-SAMs with DNA Spacer, Antibody Immobilization}

Electrodes were activated by plasma treatment. A drop of $0.05 \mu \mathrm{L}$ ethanolic solution of 0.033 mM SH-ssDNA (5'-thiol-C6-TTT TTT TTTTCC TGC GTC GTT TAA GGA AGT AC-3', purchased from Metabion, Germany) and $13.3 \mathrm{mM}$ 1,4-benzenedithiol dissolved in ethanol was pipetted on the working electrode and incubated overnight at $6{ }^{\circ} \mathrm{C}$. After rinsing with ethanol, the counter electrode was again plasma-activated while the working electrode was covered with impermeable polystyrene. $10 \mu \mathrm{L}$ of a solution of $20 \mathrm{mM} \mathrm{1,4-benzenedithiol} \mathrm{dissolved} \mathrm{in} \mathrm{ethanol} \mathrm{was} \mathrm{pipetted} \mathrm{on} \mathrm{the} \mathrm{working}$ electrode and incubated overnight at $6{ }^{\circ} \mathrm{C}$. After that, $0.05 \mu \mathrm{L}$ amino-ssDNA (5'-amino-C6- GTA CTT CCT TAA ACG ACG CAG G-3', purchased from Metabion, Germany), which was diluted with phosphate buffer to a concentration of $0.1 \mathrm{mM}$, was pipetted onto the working electrode and incubated overnight at $6^{\circ} \mathrm{C}$. To convert the amino groups to carboxyl groups for antibody coupling, glutaric anhydride was dissolved in $8 \mathrm{M}$ sodium hydroxide solution at a concentration of $0.5 \mathrm{mg} / \mu \mathrm{L}$ [11]; and $10 \mu \mathrm{L}$ of this solution was applied on the electrodes. The glutaric anhydride was supposed to react only with the amino groups of the functionalized working electrode. After $48 \mathrm{~h}$ of incubation at $6{ }^{\circ} \mathrm{C}$ the chip was rinsed with bi-distilled water. The following protocol of antibody coupling via EDC/NHS mixture and subsequent bonding of the PDMS channel was the same as described in the section before (Section 2.2.1).

\subsection{Measurements with the Microfluidic Impedance Biosensor Chip}

\subsubsection{Measurement Setup}

Impedance measurements were performed with the IMPSPEC device from Meodat (Germany), which was designed for fast and broadband impedance spectroscopy. A frequency of $5.859 \mathrm{~Hz}$ was set as starting frequency and as frequency intervals which were added stepwise until the final frequency of around $10 \mathrm{kHz}$ was reached, i.e., a linear frequency increase was applied. For each frequency, real part and imaginary part of the impedance were displayed. The impedance biosensor chip was connected to the IMPSPEC device cable by means of crocodile clamps. A peristaltic pump delivered the liquid samples through the PDMS channel and across the electrodes.

\subsubsection{HSA Adsorption for Testing SAMs}

PBS redox was prepared by adding potassium hexacyanoferrate(II) and potassium hexacyanoferrate(III) to PBS to a final concentration of $15 \mathrm{mM}$ each. HSA was dissolved in PBS redox at concentrations of 0/1/10/100/1000 ng/mL. Starting with a zero sample containing PBS redox only and corresponding to HSA, $0 \mathrm{ng} / \mathrm{mL}$, the samples were subsequently applied with increasing HSA concentration on the impedance biosensor chip. Each sample was applied for $2 \mathrm{~min}$ at a flow rate of $0.04 \mathrm{~mL} / \mathrm{min}$. After sample application, the pump was stopped, and after $30 \mathrm{~s}$ the impedance measurement was performed before the next sample was applied. 


\subsubsection{HSA Adsorption and Troponin I Assay}

A biosensor impedance chip with freshly prepared antibody coating (see Section 2.2) and bonded PDMS microfluidic channel (see Section 2.1.2) was rinsed with PBS redox for 2 min at a flow rate of $0.04 \mathrm{~mL} / \mathrm{min}$. After switching off the pump and waiting for $30 \mathrm{~s}$ the impedance of the antibodycoated biosensor chip was measured. After that, HSA dissolved at $1000 \mathrm{ng} / \mathrm{mL}$ in PBS was applied on the biosensor chip for $3 \mathrm{~min}$ at $0.04 \mathrm{~mL} / \mathrm{min}$, followed by PBS redox for 2 min at the same flow rate. The pump was stopped, and after $30 \mathrm{~s}$ the effect of HSA on the respective biosensor impedance was measured. Finally, troponin I dissolved a $1 \mathrm{ng} / \mathrm{mL}$ in PBS was applied on the biosensor chip for $1 \mathrm{~min}$ at $0.04 \mathrm{~mL} / \mathrm{min}$, followed by PBS redox for $2 \mathrm{~min}$ at the same flow rate. The pump was stopped, and after $30 \mathrm{~s}$ the impedance resulting from troponin I binding on the respective biosensor chip was measured.

\section{Results and Discussion}

\subsection{Troponin I Assay Using Thiol-SAMs Based on Hydrocarbon Spacer}

SAMs on working and counter electrode are required to minimize non-specific protein binding on the electrode surface, as this would lead to false positive signals. Furthermore, the SAM on the working electrode needs to provide functional groups for immobilizing the analyte-specific antibody. To test the performance of potential SAMs both working and counter electrode were coated with the respective thiol compound. In this work, thiols with aromatic hydrocarbon spacers were chosen to reduce the initial impedance compared to aliphatic hydrocarbon spacers [6] and, hence, to increase the permeability of the sensing layer. After the SAM coating, samples containing increasing concentrations of HSA were successively applied on the electrodes. The results are shown in Figure 3.
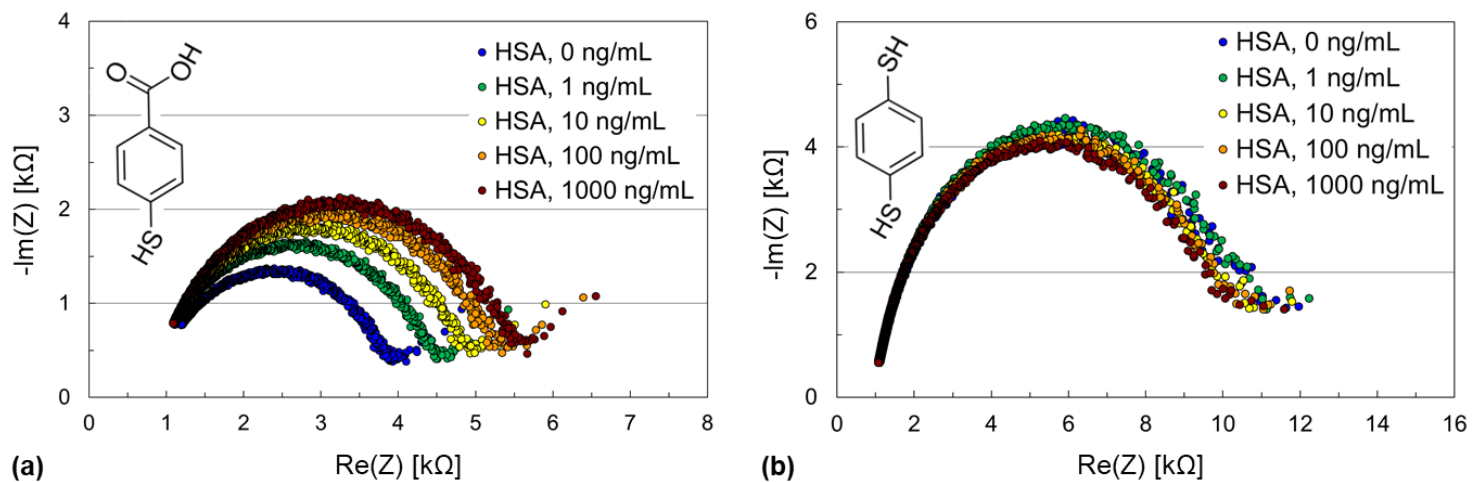

Figure 3. Nyquist plots of an impedance biosensor chip for testing SAMs with HSA samples. Both working and counter electrode were coated with (a) 4-mercaptobenzoic acid or (b) 1,4-benzenedithiol. Samples containing HSA in increasing concentrations were applied subsequently on the biosensor chip.

Both SAMs showed almost ideal semicircles except for a few frequencies at the beginning, i.e., the permeability of the SAM layer is sufficient. The initial impedance obtained with the 4mercaptobenzoic acid SAM was $4.9 \mathrm{k} \Omega$, after HSA adsorption it was $6.6 \mathrm{k} \Omega$. With 1,4-benzenedithiol an initial impedance of $12 \mathrm{k} \Omega$ was obtained with the SAM. This was higher than obtained with 4mercaptobenzoic acid, but it remained in this range after HSA adsorption. This allows the conclusion that the density of the 1,4-benzenedithiol is higher than that of the 4-mercaptobenzoic acid SAM in a way that only a negligible amount of HSA was adsorbing on the electrodes. In contrast to that, the 4mercaptobenzoic acid SAM allowed non-specific HSA adsorption, i.e., this SAM provided a lower density. However, functional groups, such as the carboxyl groups of the 4-mercaptobenzoic acid, are required for immobilization of the antibody; it is simpler than immobilization via the thiol group. Furthermore, additional immobilization steps could increase the density of the sensing layer in a way 
that non-specific HSA adsorption would be reduced to a greater extent. For that reason, experiments with 4-mercaptobenzic acid as SAM were continued. Figure 4 shows the results obtained.

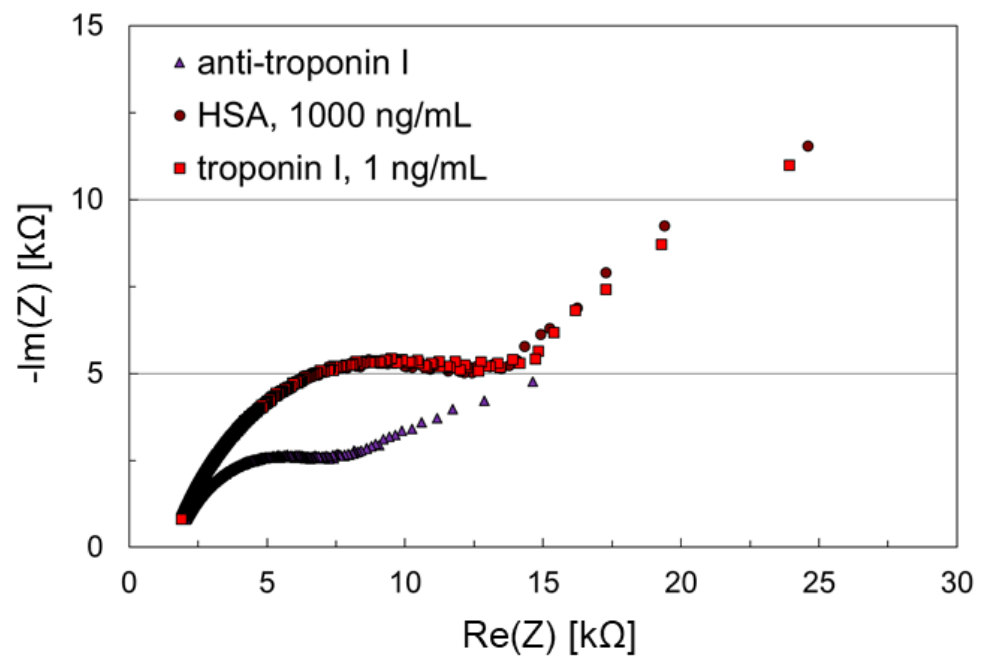

Figure 4. Nyquist plots of an impedance biosensor chip with aromatic hydrocarbon-based SAM for troponin I detection. The working electrode was coated with anti-troponin I, which was immobilized on a SAM consisting of 4-mercaptobenzoic acid. The counter electrode was coated with 1,4benzenedithiol. Samples containing HSA, $1000 \mathrm{ng} / \mathrm{mL}$, and troponin I, $1 \mathrm{ng} / \mathrm{mL}$, were applied subsequently on the biosensor chip.

The initial impedance after antibody coating was $15.4 \mathrm{k} \Omega$. Antibody immobilization, however, did not reduce HSA adsorption. After applying $1000 \mathrm{ng} / \mathrm{mL}$ the initial impedance almost doubled (27 $\mathrm{k} \Omega$ ) and remained there after troponin I was applied. Even worse, the Warburg impedance indicating diffusion of the charge carriers was increased, demonstrated by the linear ranges in the Nyquist plots for frequencies up to $100 \mathrm{~Hz}$. In this range, diffusion effects prevailed, and the layer was too insulating for observing charge transfer kinetics. Furthermore, application troponin I did not result in another signal shift, i.e., troponin I detection was not possible, at least not at that concentration.

Using hydrocarbon spacers with larger chain lengths would be favorable regarding prevention of non-specific protein adsorption, but it would also increase the impermeability of the sensing layer, hindering the charge transfer. As wet chemistry methods are preferred due to the easier coating chemistry, conducting polymers are not discussed here. Instead, oligonucleotide spacers were tested, as described in the following.

\subsection{Troponin I Assay Using Thiol-SAMs Based on DNA Spacer}

DNA strands represent large chains with negative backbone, promising a lower initial impedance. The SAM was formed by ssDNA carrying a thiol group. After hybridization with the complementary ssDNA carrying an amino group, functional groups for antibody immobilization were available (details see Section 2.2.2). An impedance biosensor chip with this coating was used for testing HSA adsorption and troponin I detection, the results are shown in Figure 5. 


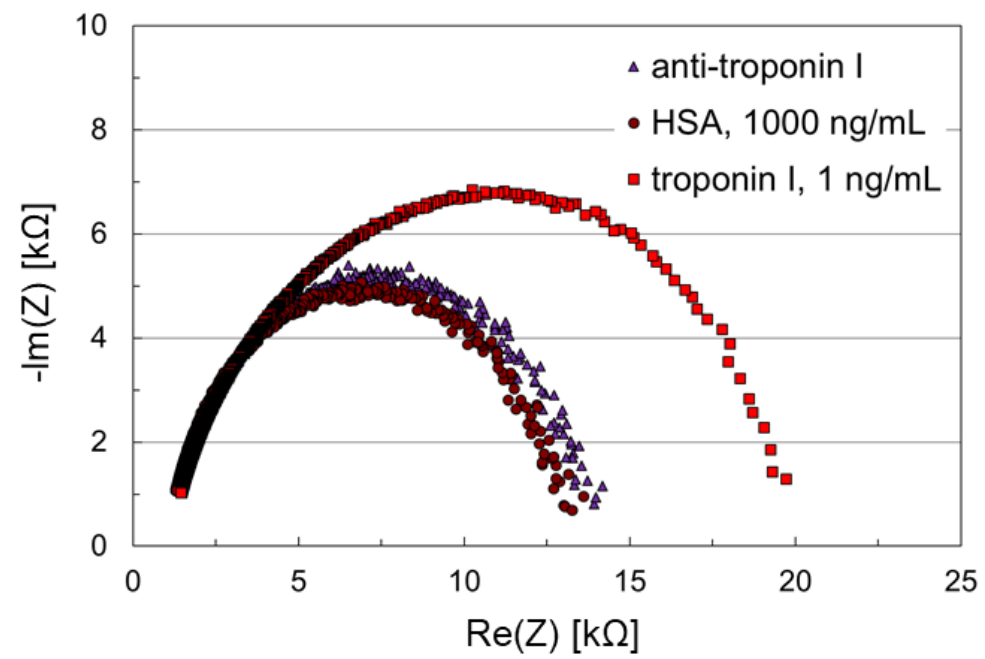

Figure 5. Nyquist plots of an impedance biosensor chip with DNA-based SAM for troponin I detection. The working electrode was coated with anti-troponin I, which was immobilized on a SAM consisting of thiolated DNA and 1,4-benzenedithiol. The counter electrode was coated with 1,4benzenedithiol. Samples containing HSA, $1000 \mathrm{ng} / \mathrm{mL}$, and troponin I, $1 \mathrm{ng} / \mathrm{mL}$, were applied subsequently on the biosensor chip.

With DNA-based SAMs, an initial impedance of only $14.2 \mathrm{k} \Omega$ was obtained, i.e., similar to the value obtained with 4-mercaptobenzoic acid SAM. However, the DNA-based SAM allowed only insignificant HSA adsorption, as shown by the low shift in the Nyquist plot and the almost identical impedance of $13.6 \mathrm{k} \Omega$. Furthermore, in contrast to Figure 4, no diffusion-predominated areas are shown in Figure 5, confirming the improved charge transfer permeability of the sensing layer using a DNA-based SAM. Application of $1 \mathrm{ng} / \mathrm{mL}$ troponin I led to a significant signal shift and an impedance of $19.8 \mathrm{k} \Omega$, but still no predominating Warburg impedance. This shows that DNA-based SAMs are most promising for sensing layers of impedance biosensor chips.

\section{Conclusions}

A microfluidic impedance biosensor chip was developed allowing the determination of $1 \mathrm{ng} / \mathrm{mL}$ cardiac biomarker troponin I. The use of thiols with DNA spacer showed excellent results regarding both reduction of non-specific protein adsorption (HSA) and detection of low concentrations of biomarker (troponin I). Sampling and measurement took only a few minutes, whereby the main part of the time was taken up by the sampling. The results were promising regarding a future costeffective biosensor array chip for the rapid detection of clinically relevant biomarkers in real samples, such as serum and saliva.

Author Contributions: Conceptualization, T.R., S.S., K.L.; data curation, K.L.; formal analysis, K.A., T.H., K.L.; investigation, K.A., T.H.; methodology, T.R., S.S., A.V., K.L.; project administration, T.R., S.S., K.L.; resources, T.R., K.L.; supervision, T.R., K.L.; validation, K.A., K.L.; visualization, K.A., T.H., K.L.; writing - original draft preparation, K.L.; writing - review and editing, K.L. All authors have read and agreed to the published version of the manuscript.

Funding: This research received no external funding.

Conflicts of Interest: The authors declare no conflict of interest.

\section{References}

1. Cardiovascular Diseases (CVDs). WHO Fact Sheet 2017. Available online: https://www.who.int/en/newsroom/fact-sheets/detail/cardiovascular-diseases-(cvds) (accessed on 24 September 2020). 
2. Taylor, C.J.; Ordóñez-Mena, J.M.; Roalfe, A.K.; Lay-Flurrie, S.; Jones, N.R.; Marshall, T.; Hobbs, F.D.R. Trends in survival after a diagnosis of heart failure in the United Kingdom 2000-2017: Population based cohort study. BMJ 2019, 364, 1223.

3. Dörner, K. Klinische Chemie und Hämatologie, 7th ed.; Georg Thieme Verlag: Stuttgart, Germany, 2009.

4. Luppa, P.B.; Junker, R. (Eds.) Point-of-Care Testing: Principles and Applications, 1st ed.; Springer-Verlag GmbH: Berlin, Germany, 2018.

5. Gruhl, F.J.; Rapp, B.E.; Länge, K. Biosensors for diagnostic applications. Adv. Biochem. Eng. Biotechnol. 2013, 133, 115-148.

6. Pires Carneiro, L. Development of an Electrochemical Biosensor Platform and a Suitable Low-Impedance Surface Modification Stategy; KIT Scientific Publishing: 2014.

7. Leva-Bueno, J.; Peyman, S.A.; Millner, P.A. A review on impedimetric immunosensors for pathogen and biomarker detection. Med. Microbiol. Immunol. 2020, 209, 343-362.

8. Steel, A.B.; Levicky, R.L.; Herne, T.M.; Tarlov, M.J. Immobilization of nucleic acids at solid surfaces: Effect of oligonucleotide length on layer assembly. Biophys. J. 2000, 79, 975-981.

9. Rapp, B.E.; Voigt, A.; Dirschka, M.; Länge, K. Deposition of ultrathin parylene C films in the range of 18 $\mathrm{nm}$ to $142 \mathrm{~nm}$ : Controlling the layer thickness and assessing the closeness of the deposited films. Thin Solid Films 2012, 520, 4884-4888.

10. Länge, K.; Gruhl, F.J.; Rapp, M. Surface acoustic wave (SAW) biosensors: Coupling of sensing layers and measurement. Methods Mol. Biol. 2013, 949, 491-505.

11. Länge, K.; Gruhl, F.J.; Rapp, M. Influence of preparative carboxylation steps on the analyte response of an acoustic biosensor. IEEE Sens. J. 2009, 9, 2033-2034.

Publisher's Note: MDPI stays neutral with regard to jurisdictional claims in published maps and institutional affiliations.

(C) 2020 by the authors. Submitted for possible open access publication under the terms and conditions of the Creative Commons Attribution (CC BY) license (http://creativecommons.org/licenses/by/4.0/). 